Proc. 15th Int. Conference on Defects Recognition, Imaging and Physics in Semiconductors, Warsaw, Poland 2013

\title{
Focused Ion Beam Imaging of Defects in Multicrystalline Si for Photovoltaic Application
}

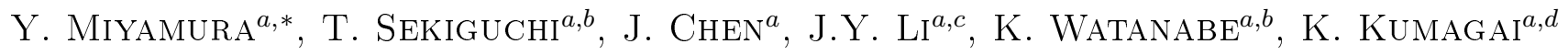 \\ AND A. OGURA ${ }^{c}$ \\ ${ }^{a}$ MANA Nanoelectronic Materials Unit, National Institute for Materials Science \\ 1-1 Namiki, Tsukuba, Ibaraki 305-0044, Japan \\ ${ }^{b}$ Graduate School of Pure and Applied Sciences, University of Tsukuba \\ 1-1 Namiki, Tsukuba, Ibaraki 305-0044, Japan \\ ${ }^{c}$ School of Science and Technology, Meiji University, Kawasaki 214-8571, Japan \\ ${ }^{d}$ National Metrology Institute of Japan, National Institute of Advanced Industrial Science and Technology \\ 1-1-1 Higashi, Tsukuba, Ibaraki 305-8565, Japan
}

\begin{abstract}
We demonstrate the imaging of the extended defects in Si materials using a focused ion beam instrument. Since Ga-ion beam has small penetration depth and high channeling character compared with electron beam, the secondary electron signal of focused ion beam is more sensitive to the surface morphology and crystallinity. We have tried to use this secondary electron imaging of focused ion beam for observation of various extended defects in Si materials for photovoltaic and semiconductor devices. As for the texture of multicrystalline Si, some grains are imaged darker than the others. It suggests that the crystal orientation gives different channeling effect on the primary Ga-ion beam, resulting in the different secondary electron yield. The grain boundaries and lineage in multicrystalline $\mathrm{Si}$ are shown as bright lines and patterns in the image. Although it may reflect the surface morphologies, these contrasts may be attributed to the channeling contrast due to lattice displacement or distortion. The contrast mechanism of FIB imaging is discussed.
\end{abstract}

DOI: 10.12693/APhysPolA.125.991

PACS: 61.05.-a, 61.85.+p, 61.72.Ff

\section{Introduction}

The development of focus ion beam (FIB) has widened the possibility of electron microscope because it enables us to pick up the position of interest and fabricate proper specimens for observation. In addition to this advantage, FIB has a potential for the Ga-ion beam microscope. The early FIB possesses both Ga-ion source for fabrication and electron beam source for observation. The recent model only has one Ga-ion gun not only for fabrication but also observation. Such FIB has wide range of Ga-ion beam current and the secondary electron (SE) detector was customized for collecting SEs generated by Ga-ion injection.

There exist many pioneering works on the scanning ion microscopy (SIM) [1-3]. Among these works, grain imaging of metals and alloys, corrosion studies were highlighted due to their superior imaging of these materials $[4,5]$. On the other hand, the application of semiconductor was mostly focused on the FIB processing and not so much effort has been done for defect imaging. In this study, we have elucidated the possibility of the defect imaging in Si samples.

*corresponding author; e-mail: miyamura.yoshiji@nims.go.jp

\section{Experimental}

FIB (JIB-4000, JEOL Co. Ltd, Japan) was used for SIM imaging. This instrument is composed of a Ga-ion gun and an SE detector. The Ga-ion beam voltage and current are widely variable, from 1 to $30 \mathrm{kV}$, and from $1 \mathrm{pA}$ to $60 \mathrm{nA}$, respectively. All the images were taken with SE signals induced by Ga-ion irradiation. This image is called Ga-ion induced secondary electron (abbreviated as GaISE), hereafter. The observation was done for the multicrystalline (mc-) Si for photovoltaic application grown by cast method $[6,7]$.

\section{Results and discussion}

Figure 1 shows the GaISE images of mc-Si specimen taken at a Ga-ion beam of $20 \mathrm{kV}$ and $20 \mathrm{pA}$. Different grains are imaged as dark or bright regions. The rectangles at the central grains are the lamellae of twins in this grain. Some bright patterns exist in the right area, indicating that some extended defects are visible in this GaISE image.

Figure 2 shows the GaISE images of different part of mc-Si specimen taken at a Ga-ion beam of $30 \mathrm{kV}$ and 200 pA. Contrary to Fig. 1, the grain boundaries (GBs) are highlighted as bright lines in these images. The grain contrast is overlapped with this GB contrast. As is seen in Fig. 2b, it should be noted that not only GB but also 


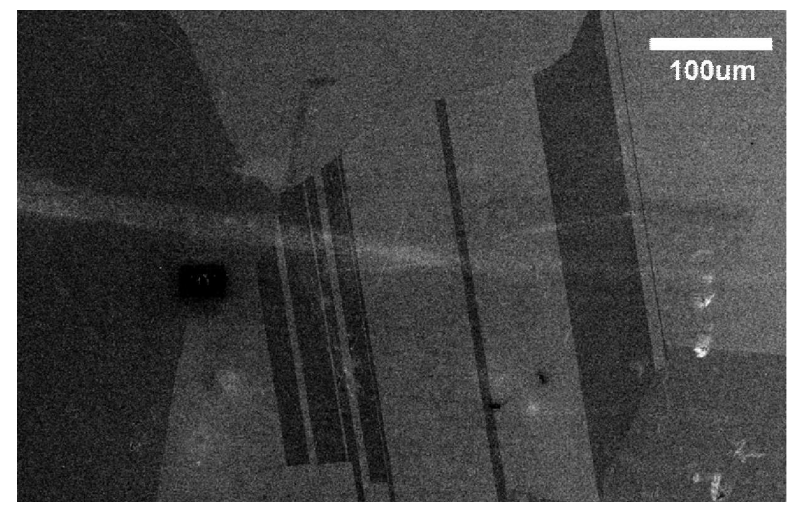

Fig. 1. GaISE image of mc-Si wafer $(20 \mathrm{kV}, 20 \mathrm{pA})$.

dislocations are visible. The brightness of GBs and dislocations varies on the observing conditions, not only on the Ga-ion beam but also scan speed. Generally, the higher ion beam energy and higher current give the brighter contrast. It should be noted that not all the GBs are bright, but some are dark.

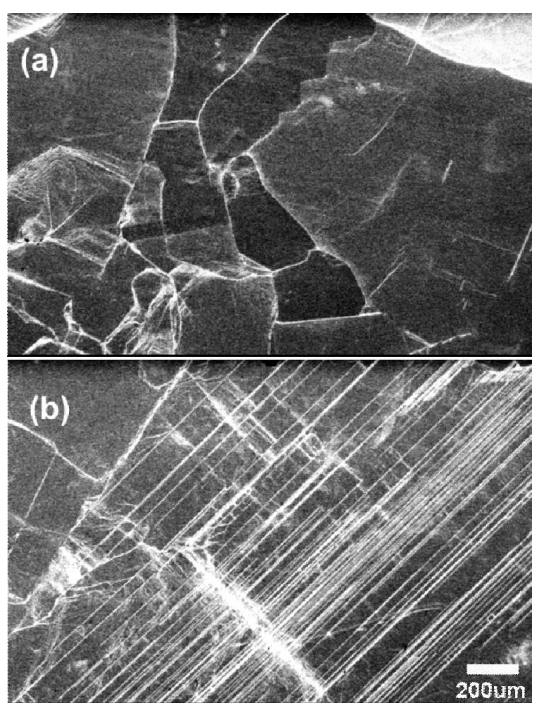

Fig. 2. GaISE images of mc-Si wafer $(30 \mathrm{kV}, 200 \mathrm{pA})$.

Figure 3 shows an area including straight bright GB and white lamellae defects. The dark GB may be seen due to the deep groove.

The white lamellae is seen as the row of short white segments as shown in Fig. 4. These contrasts have not changed by the 90 degree specimen rotation. This indicates that these contrasts are not related to the geometrical configuration between the defect and SE detector.

Figure 5 shows the GaISE images of the same area as Fig. 3 taken with the different Ga-ion energies. Although the straight GB is seen dark in all the images, the lamellae are dark at $1 \mathrm{kV}$ and turn to bright at higher energies above $15 \mathrm{kV}$.

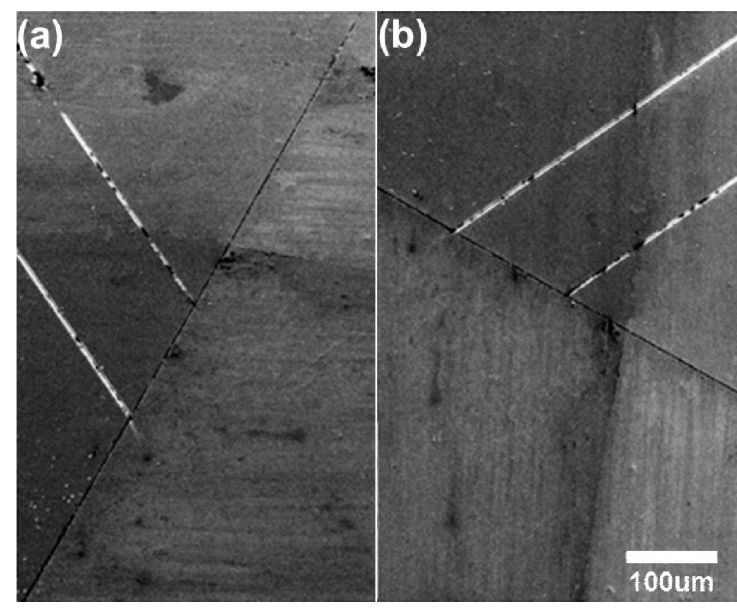

Fig. 3. GaISE image of mc-Si wafer. Specimen rotation angles are (a) 0 degree and (b) 90 degree.

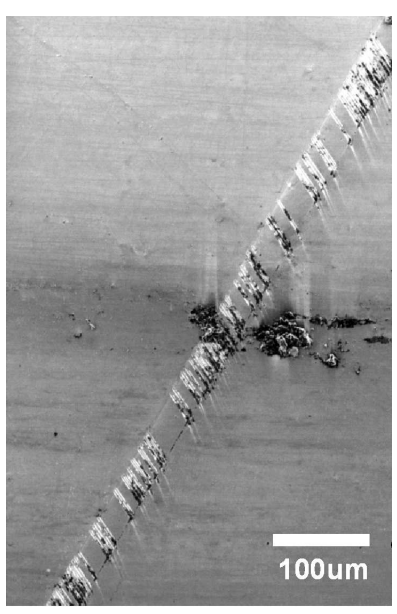

Fig. 4. GaISE image of mc-Si wafer.

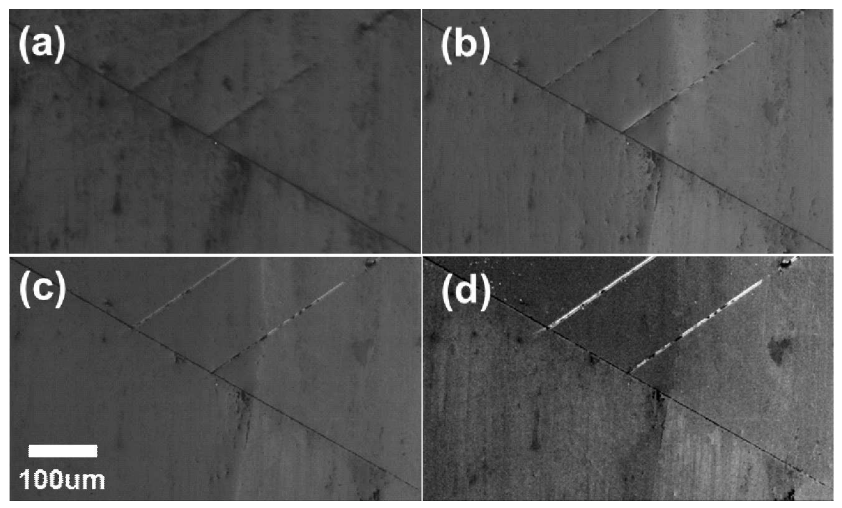

Fig. 5. GaISE images of mc-Si wafer. Ga-ion energies were (a) $1 \mathrm{kV}$, (b) $5 \mathrm{kV}$, (c) $15 \mathrm{kV}$, and (d) $30 \mathrm{kV}$. 
These observations give the possibility of GaISE for the defect observation of mc-Si. GaISE image gives us the information of grains and GB/dislocations.

As for the grains, GaISE images are not so much sensitive compared with the conventional electron beam induced SE (eISE) images. The contrast relation of grains does not change significantly by specimen tilting. It suggests that the channeling effect is not so dominant in this case. This is very different from the observation of polycrystalline metals and alloys. Such difference may be derived from the low density nature of diamond structure. The open space structure may suppress the channeling effect.

The bright contrast of GBs and dislocations may infer the effect of strain. Since the curved dislocations seem to give brighter contrast, this may be a possible explanation. However, the straight lines in Fig. $2 b$ are the $\Sigma 3 \mathrm{GBs}$, which possess small local strain variation. Another possible explanation is the surface roughness. Since the surface of this specimen is slightly etched, the vicinity of GBs and dislocations are not flat. If Ga-ion beam is sensitive to the surface morphology, this explanation may make sense. In Fig. 4, the white lines in the lamellae accompany the streak with the right side. These streaks are also recognized as the slight bumps in eISE image, which suggests that the dislocations underlay these area.

The Ga-ion energy dependence of Fig. 5 is also worthwhile to discuss. Normally, the penetration depth of Ga-ion is very small compared with electron beam. The range of $30 \mathrm{kV}$ Ga-ion is around $30 \mathrm{~nm}$, while that of electron is $9.4 \mu \mathrm{m}$. This suggests that most of GaISE has generated at the region less than $100 \mathrm{~nm}$ even taken into account of channeling. The variation of Ga-ion energy may suggest that the GaISE is very sensitive to the surface structure. At this moment, it is rather difficult to draw the reliable mechanism of GaISE imaging of extended defects of mc-Si. However, we may point out that the GaISE is very sensitive to the tiny surface morphology, especially the faint etch surface. Thus, we propose
GaISE for the enhanced imaging technique of etched surface. Hereafter, not only to study the Ga-ion beam theory but also optimize the signal detector are necessary to develop this method of defect observation.

\section{Summary}

GaISE imaging using FIB instrument was demonstrated for the defect observation in mc-Si. Grains are imaged as the different brightness in lower Ga-beam condition $(20 \mathrm{kV}$ and $20 \mathrm{pA})$. Grain boundaries and dislocations become bright in higher beam condition $(30 \mathrm{kV}$ and $200 \mathrm{pA}$ ). These contrast mechanisms were discussed. At this stage, we may conclude that GaISE is extremely sensitive to the slightly etched mc-Si surface.

\section{Acknowledgments}

This work was partly supported by the New Energy and Industrial Technology Development Organization (NEDO) under the Ministry of Economy, Trade and Industry (METI).

\section{References}

[1] M.W. Phanehf, Micron 30, 277 (1999).

[2] J. Li, Mater. Lett. 62, 804 (2008).

[3] D.L. Barr, W.L. Brown, J. Vac. Sci. Technol. B 13 2580 (1995).

[4] T. Suzuki, N. Endo, M. Shibata, S. Kamasaki, T. Ichinokawa, J. Vac. Sci. Technol. A 22, 49 (2004).

[5] M.D. Uchic, M.A. Groeber, D.M. Dimiduk, J.P. Simmons, Scr. Mater. 55, 23 (2006).

[6] K. Arafune, E. Ohishi, H. Sai, Y. Ohshita, M. Yamaguchi, J. Cryst. Growth 308, 5 (2007).

[7] K. Jiptner, M. Fukuzawa, Y. Miyamura, H. Harada, K. Kakimoto, T. Sekiguchi, Phys. Status Solidi C 10, 141 (2013). 